\title{
Lacunae regarding dearth of dissection-based teaching during COVID-19 pandemic: how to cope with it?
}

\author{
Sanjib Kumar Ghosh ${ }^{1}$ (D)
}

Received: 19 June 2021 / Accepted: 16 August 2021 / Published online: 20 August 2021

(c) The Author(s), under exclusive licence to Springer-Verlag France SAS, part of Springer Nature 2021

\begin{abstract}
Introduction In view of the ongoing COVID-19 pandemic, anatomy education programs were amended so as to shift to primarily online mode from physical classes. In the whole process, main concern area that has emerged is regarding dearth of physical human dissection sessions. There is enough evidence available in literature to suggest that dissection room is an ideal place to cultivate and inculcate discipline independent skills or humanistic skills among the students. These include attributes in relation to ethical practice, professionalism, communication skills, empathy and compassion. Imbibing these skills are an essential element of medical education curriculum as it is desirable that students exhibit these traits when they begin medical practice. Hence deficiency in terms of exposure to physical dissection sessions can adversely affect training of medical students on a long term.

Methods A literature search of relevant, peer-reviewed, published articles was undertaken from indexed databases (Medline and PubMed, Scopus, Embase, CINAHL Plus, Web of Science and Google Scholar) for this study.

Results To counter the pertinent issue in online anatomy teaching program, a few measures have been suggested in this paper based on identification of actual deficit areas in terms of learning and analysis thereof. Live streaming of real time dissection, awareness sessions on human dissection, online interactive learning sessions and reflective thoughts-based exercise can contribute to building discipline independent skills in present scenario.

Conclusion Incorporation and implementation of these interventions within the realm of online anatomy education programs during COVID-19 pandemic can possibly contribute towards desirable learning outcomes.
\end{abstract}

Keywords COVID-19 pandemic · Online classes · Human dissection · Humanistic skills · Intervention · Learning outcome

\section{Shortfall of physical human dissection sessions during COVID-19 pandemic (problem statement)}

The ongoing COVID-19 pandemic has adversely affected the domain of medical education across the globe for the last one and half years. Similar to all disciplines of medicine, the anatomy curriculum had to adapt itself in accordance with the prevailing circumstances [7]. The primary challenge for anatomists has been to shift the teaching medium from physical to online mode. Such a transition became inevitable for medical schools around the world and necessary

Sanjib Kumar Ghosh

drsanjib79@gmail.com

1 Department of Anatomy, All India Institute of Medical Sciences, 3rd Floor, Academic Building, Phulwari Sharif, Patna, Bihar 801507, India measures had to be undertaken within a short notice [3]. Within the realm of anatomical science, the main concern area was the exercise of human dissection. In most medical schools, human dissection constitutes the core element of teaching/learning anatomy [10]. For an anatomy educator who is used to teach physically through human dissection, the very idea of online demonstration appears to be no less than claustrophobic. This is attributed to deficiency in terms of physical exposure to anatomical specimens (human tissues) and consequent lack of tactile/ experiential learning which is associated with human dissection [17]. In general, the perception is that online model of teaching anatomy from human body has serious limitations [6]. By the turn of twenty-first century, educators have realized that human dissection is a unique learning medium within the medical education curriculum. It no longer can be identified as a solely knowledge-based exercise as it has been over the years since its inception. Rather, human dissection has evolved as 
a potent medium for cultivating and inculcating discipline independent skills such as professionalism, ethics, compassion and communication skills among medical students $[12,13,15,16]$. In a way, the dissection room trains a budding future physician towards being a compassionate and empathetic clinician of tomorrow. In other words, the act of human dissection during anatomy curriculum imparts the first lesson to medical students for being a physician with desirable humanistic traits, which are so very essential to serve the society with a tender healing touch [1]. Hence it may be opined that in recent years, prior to the onset of pandemic, human dissection evolved as an essential as well as critical component of medical education owing to its unique role in cultivating humanistic traits among medical students at an early stage of their career. However, due to unforeseen circumstances and with the overwhelming dominance of online (digital) medium in the midst of a rampaging pandemic, human dissection (in its true physical form) has taken a backseat in anatomy education [20]. Consequently, the training of medical students in discipline independent skills or humanistic skills have also suffered. Although human dissection is associated with inherent drawbacks and educators have suggested effective replacements [2, 4], nevertheless as educators it is pertinent to realize that a compromise in terms of desirable outcome of dissection-based learning during COVID-19 pandemic can adversely affect the eventual fabric of the medical students when they are licensed to undertake medical practice [9]. Hence judicious analysis of the current situation and logical remedial measures are needed to minimize the process of attrition. In this study, attempt will be made to identify the principal deficit areas in terms of online anatomy teaching as compared to physical human dissection-based learning (lacunae due to dearth of dissection-based teaching). This will be followed by suggested measures (coping mechanism) which can potentially act as a makeshift model for imparting humanistic skills to medical students till the time anatomy education reverts back to traditional human dissection-based learning methods.

\section{Methods}

The study was conducted in the Department of Anatomy at All India Institute of Medical Sciences, Patna, India. An extensive literature search was undertaken for this study from the following indexed databases:

1. Medline and PubMed (United States National Library of Medicine, Bethesda, MD).

2. Scopus (Elsevier, Amsterdam, The Netherlands).

3. Embase (Ovid Technologies, Inc., New York, NY).

4. CINAHL Plus (EBSCO Information Services, Ipswich, MA).
5. Web of Science (Clarivate Analytics, Philadelphia, PA).

6. Google Scholar (Google, Inc., Mountain View, CA).

The literature search was based on key terms relevant to the topic of the present study. Accordingly, the following terms were used during literature search: "anatomy education during COVID-19; "human dissection during COVID19"; "role of human dissection in medical education"; "online teaching strategies in anatomy"; "online teaching methods during COVID-19" and "impact of COVID-19 on anatomy education". The literature search was limited to only those studies with relevant information. After completion of literature search, the findings were compiled and final observations were prepared.

\section{Lacunae created in terms of learning outcome due to dearth of dissection sessions}

Deficiency in terms of not having physical human dissection sessions amidst the ongoing COVID-19 pandemic, adversely affects four areas of student learning:

A. The foremost among them is the very act of human dissection. While undertaking human dissection, every student goes through a process of silent interaction with the mortal remains of a human being who was once alive. At some point of time, students realize that the deceased person is contributing to advancing his/her knowledge in anatomy by going under the knife and thereby doing a noble service to the society at large. This altruistic act of the person whose mortal remains are dissected sow the seeds of empathy and compassion among the students $[23,26]$. Eventually these attributes are exhibited when they handle live patients later on in their clinical career. In a way, the human cadaver, therefore, performs the role of the first teacher in anatomy and guides every student such that they can be better physicians of tomorrow [11].

B. The dissection room premises also provide a unique learning platform for medical students. It is the seat of inculcating a set of good practices related to ethical behaviour among medical students. These include maintaining a proper decorum (no outsiders, no loud noise, no use of mobile phones and no clicking selfies/photos), being in notified attire (wearing lab coats/ apron/ any other specified by concerned authorities), refraining from activities unbecoming of the solemn environment of the place (no eating food, no drinking beverages and not indulging in banter) and showing respect to the human cadaver being dissected (maintaining sanctity of the human remains and maintaining cleanliness in and around the field of dissection). All these daily practices 
cultivate the values of ethical practice among the students, which they are supposed to imbibe in their attitude while undertaking clinical duties [13, 14].

C. Interactions between the students is an integral part of dissection-based learning. The level of interaction may vary depending on whether dissection or prosectionbased teaching is practised [19]. But the fact that interaction takes place among students has paramount significance. This aspect constitutes a priceless opportunity to learn communication skills, which is critical to being a good physician. It is pertinent to mention here that the communication among students may not amount to reciprocal peer teaching [19]. Rather significant element of this communication process includes not making any funny/unacceptable references to the human cadaver, keeping the communication undertaken in the dissection room premises to oneself and not sharing it with a third person (family member and friends). This aspect of dissection room learning is vital towards developing skills relevant to professionalism among the students [13,21].

D. The interaction between students and their teacher/ facilitator is also a remarkable learning exercise pertaining to dissection-based teaching. These communication attributes include feedback session for the students regarding their activities and attitude during their stay in the dissection room premises. They often include reflective exercises which encourages students to delve deep into their own performance and come up with remedial measures if any or suggestions critical towards further improvement of the whole process. This aspect also promotes skills pertaining to professionalism whereby students adapt themselves to receiving feedback at different levels of their medical career and indulging in reflective exercises based on feedback received thus walking the mile towards self-improvement $[8,24]$.
Hence it is pertinent to mention that these critical components of training medical students are being compromised as physical dissection classes have come to a standstill due to the ongoing pandemic [18].

\section{Measures that can be adopted to address the issue at hand}

To overcome the deficiencies in terms of lack of dissectionbased teaching amidst COVID-19 pandemic, a few measures may be adopted as interim arrangement till the time the situation improves (Fig. 1).

1. Live online streaming of real time dissection sessions from the dissection room. It is critical to ensure that these sessions simulate the physical dissection as much as possible. This can be achieved by motivating the students to maintain the good practices (associated with a physical dissection class) during online sessions also. Hence students should attend these sessions in dissection room attire (lab coat/apron/any other approved) and should not record any videos neither they should share the links/ sessions with any outsider (family/friend/close relative). They should not resort to eating or drinking while the session is going on. Moreover, they should not make any comments which is unbecoming of the session. Implementation of this measure requires a stable internet connection at both ends, which at times can be a hindrance. Online streaming of virtual dissection is also an option in institutions where physical dissection is not practised. This statement is based on the outcome analysis of virtual dissection in anatomy education [25].

2. Awareness sessions on human dissection can be incorporated in the teaching/ learning roster. These online

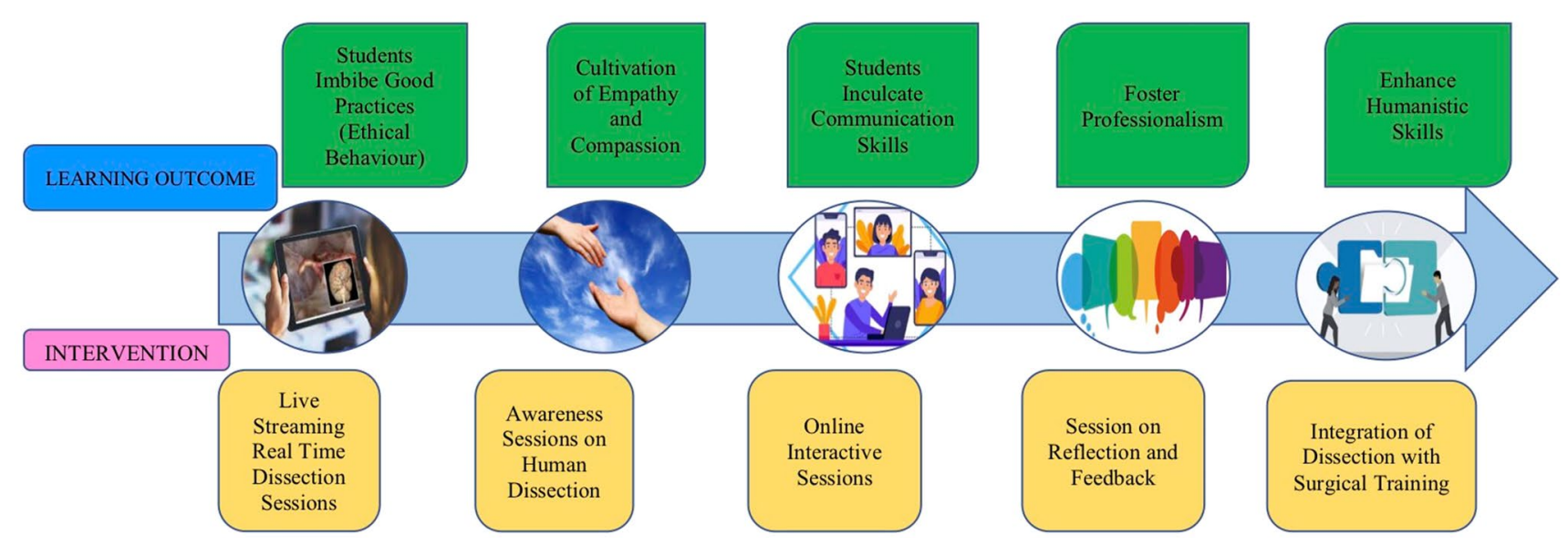

Fig. 1 Measures to cope with the dearth of human dissection-based teaching practices during COVID-19 pandemic. The images used in the figure are in public domain and free from copyright issues 
sessions are meant to sensitize the students regarding the selfless altruistic act of the person, whose body is being dissected for imparting anatomy knowledge. These sessions can also preach the students regarding various ethical measures followed in the overall process of dissection-based teaching.

3. Online interactive learning sessions involving dissection related case scenarios. These sessions will have minimal role of the facilitator other than designing the case scenarios. In a way, these sessions are quite similar to problem-based learning, but the structure needs to be designed in accordance with online environment [22]. These sessions will ensure communication between the students which will be enhanced in the process. During COVID-19 pandemic, when students are living like isolated islands, such opportunity to communicate among themselves will be embraced as a welcome gesture and will serve the purpose in cultivating appropriate professional skills.

4. Reflective thoughts-based exercise needs to be encouraged among the students based on their own experiences during sessions as detailed in points 1,2 and 3. Based on the responses provided by the students a follow up feedback session would be ideal to conclude the whole exercise on a weekly or bi-weekly basis. The insights gained from these sessions will be vital in developing the professional outlook of the students.

5. Integrating physical dissection sessions with the surgical training (which begins at a later stage of medical career) based on the understanding that the situation will improve by then so as to allow physical classes. As no amount of online classes can actually compensate the experience of actual human dissection, hence it is essential to expose the students to this unique and exclusive learning tool at some point of their career. These condensed physical sessions can possibly be used to build on the discipline independent skills the students imbibed through online sessions.

\section{Discussion}

The measures suggested in this study have mostly been analysed individually in published literature with respect to their effectiveness in achieving desirable learning outcomes as outlined in Fig. 1. It has been established that these measures when implemented within the realm of online learning environment contribute to building one or more discipline independent skills among the students. In other words, there is evidence to suggest that these measures have been linked to the established theoretical concepts of learning $[5,18$, 27]. Hence in this study, the focus was not to evaluate the effectiveness of these measures. Rather the study attempts to collate all the relevant measures pertaining to online classes for anatomy such that all possible components of discipline independent skills associated with dissection-based teaching are imparted to the students as far as possible. The suggestions proposed in this study are aimed at minimizing the adverse effects of online learning mode in anatomy regarding the acquisition of discipline independent skills (humanistic skills) by the students which under normal circumstances are imparted through the practice of human dissection in a physical environment. The contents of this study hold relevance in an overwhelmingly dominant online learning environment prevailing during ongoing COVID-19 pandemic.

\section{Conclusion}

Human dissection is an integral component of anatomy education and learning outcomes achieved through this medium cannot be replicated through any alternate strategies or measures. However, considering the ongoing COVID-19 pandemic, harsh reality is that restarting physical dissection sessions is not possible in the nearest future. Hence to overcome the deficit in terms of learning outcomes due to dearth of dissection sessions (as outlined in this study and identified as lacunae pertaining to online anatomy teaching), few interim remedial measures (coping mechanism to plug the lacunae) as detailed in this paper may be incorporated into online anatomy education programs during COVID-19 pandemic.

Acknowledgements None to mention.

Author contributions SKG designed and developed the project, collected and analysed the data, wrote and edited the manuscript and approved the final submitted version.

Funding None received.

\section{Declarations}

Conflict of interest None to report.

Ethical approval Not required for this study.

\section{References}

1. Abrams MP, Eckert T, Topping D, Daly KD (2020) Reflective writing on the cadaveric dissection experience: an effective tool to assess the impact of dissection on learning of anatomy, humanism, empathy, well -being, and professional identity formation in medical students. Anat Sci Educ. https://doi.org/10.1002/ase.2025

2. Bahsi I, Topal Z, Cetkin M, Orhan M, Kervancioglu P, Odabasioglu ME, Cihan OF (2021) Evaluation of attitudes and opinions of medical faculty students against the use of cadaver in anatomy 
education and investigation of factors affecting their emotional responses related thereto. Surg Radiol Anat 43:481-487

3. Baptiste YM (2021) Digital feast and physical famine: The altered ecosystem of anatomy education due to the Covid-19 pandemic. Anat Sci Educ. https://doi.org/10.1002/ase.2098

4. Boscolo-Berto R, Tortorella C, Porzionato A, Stecco C, Picardi EEE, Macchi V, De Caro R (2021) The additional role of virtual to traditional dissection in teaching anatomy: a randomized controlled trial. Surg Radiol Anat 43:469-479

5. Brasset C, Cosker T, Davies DC, Dockery P, Gillingwater TH, Lee TC, Milz S, Parson SH, Quondamatteo F, Wilkinson T (2020) COVID-19 and anatomy: stimulus and initial response. J Anat 237:393-403

6. Byrnes KG, Kiely PA, Dunne CP, McDermott KW, Coffey JC (2021) Communication, collaboration and contagion: "virtualization" of anatomy during COVID-19. Clin Anat 34:82-89

7. Evans DJR, Bay BH, Wilson TD, Smith CF, Lachman N, Pawlina W (2020) Going virtual to support anatomy education: a STOPGAP in the midst of the Covid-19 pandemic. Anat Sci Educ 13:279-283

8. Evans DJR, Pawlina W (2021) Effects of Covid-19: The need to assess the real value of anatomy education. Anat Sci Educ 14:129-131

9. Franchi T (2020) The impact of the Covid-19 pandemic on current anatomy education and future careers: a student's perspective. Anat Sci Educ 13:312-315

10. Ghosh SK (2017) Cadaveric dissection as an educational tool for anatomical sciences in the 21 st century. Anat Sci Educ 10:286-299

11. Ghosh SK (2017) Paying respect to human cadavers: We owe this to the first teacher in anatomy. Ann Anat 211:129-134

12. Ghosh SK, Kumar A (2019) Building professionalism in human dissection room as a component of hidden curriculum delivery: a systematic review of good practices. Anat Sci Educ 12:210-221

13. Ghosh SK (2020) The practice of ethics in the context of human dissection: setting standards for future physicians. Ann Anat 232:151577

14. Ghosh SK (2020) Transformation of the role of human dissection in medical education: Cultivating principles of medical ethics. Surg Radiol Anat 42:855-856

15. Goss AL, Viswanathan VB, Delisser HM (2019) Not just a specimen: a qualitative study of emotion, morality and Professionalism in one medical school gross anatomy laboratory. Anat Sci Educ 12:349-359

16. Kaye A, Miranda M, Jones T (2019) The donor letter project: learning professionalism and fostering empathy in an anatomy curriculum. J Med Humanit 40:607-612
17. Kelsey AHCM, McCulloch V, Gillingwater TH, Findlater GS, Paxton JZ (2020) Anatomical sciences at the University of Edinburgh: initial experiences of teaching anatomy online. Transl Res Anat 19:100065

18. Kim JW, Myung SJ, Yoon HB, Moon SH, Ryu H, Yim JJ (2020) How medical education survives and evolves during COVID-19: our experience and future direction. PLoS ONE 18:e0243958

19. Lackey-Cornelison WL, Bauler LD, Smith J (2020) A comparison of the effectiveness of dissection and prosection on short-term anatomic knowledge retention in a reciprocal peer-teaching program. Adv Physiol Educ 44:239-246

20. Longhurst GJ, Stone DM, Dulohery K, Scully D, Campbell T, Smith CF (2020) Strength, weakness, opportunity, threat (SWOT) analysis of the adaptations to anatomical education in the United Kingdom and Republic of Ireland in response to the Covid-19 pandemic. Anat Sci Educ 13:301-311

21. Manyama M, Stafford R, Mazyala E, Lukanima A, Magele N, Kidenya BR, Kimwaga E, Msuya S, Kauki J (2016) Improving gross anatomy learning using reciprocal peer teaching. BMC Med Educ 22:95

22. Ng ML, Bridges S, Law SP, Whitehill T (2014) Designing, implementing and evaluating an online problem-based learning (PBL) environment- a pilot study. Clin Linguist Phon 28:117-130

23. Pais D, Casal D, Mascarenhas-Lemos L, Barata P, Moxham BJ, Goyri-O'Neill J (2017) Outcomes and satisfaction of two optional cadaveric dissection courses: A 3 -year prospective study. Anat Sci Educ 10:127-136

24. Shiozawa T, Glauben M, Banzhaf M, Griewatz J, Hirt B, Zipfel S, Lammerding-Koeppel M, Herrmann-Werner A (2020) An insight into professional identity formation: qualitative analyses of two reflection interventions during the dissection course. Anat Sci Educ 13:320-332

25. Tretter JT, Gupta SK, Izawa Y, Nishii T, Mori S (2020) Virtual dissection: emerging as the gold standard of analysing living heart anatomy. J Cardiovasc Dev Dis 7:30

26. Tseng W, Lin Y (2016) Detatched concern of medical students in a cadaver dissection course: a phenomenological study. Anat Sci Educ 9:265-271

27. Yoo H, Kim D, Lee YM, Rhyu IJ (2021) Adaptations in anatomy education during COVID-19. J Korean Med Sci 36:e13

Publisher's Note Springer Nature remains neutral with regard to jurisdictional claims in published maps and institutional affiliations. 